\title{
ELECTRODYNAMIC INSTABILITY OF A SELF-GRAVITATING FLUID CYLINDER UNDER GENERAL VARYING ELECTRIC FIELD
}

\author{
A.E. RADWAN \\ Department of Mathematics, Faculty of Science, Ain-Shams University \\ Abbassia, Cairo, Egypt
}

AND A.R.F. ELIIEFNAWY

Department of Mathematics, Faculty of Science, Banha University, Banha, Egypt

(Received May 25, 1990)

The electrodynamic instability of a self-gravitating dielectric fluid penetrated by a uniform axial clectric ficld surrounded by a self-gravitating vacuum pervaded by general varying electric field is investigated. A general eigenvalue relation valid to all possible (symmetric and asymmetric) modes of perturbation for all (short and long) wavelengths is derived and discussed in detail. The model is gravitationally stable to the pure asymmetric disturbances modes while to symmetric modes it is so if the longitudinal wave number normalized with respect to the jet radius is equal to or greater than 1.0668 and vice versa. The axial electric fields pervaded interior and exterior to the cylinder are stabilizing or destabilizing for all disturbance modes according to some restrictions. The transverse varying electric field is purely stabilizing in the symmetric disturbances for all wavelengths, while it is stabilizing in the asymmetric disturbance under some restrictions. The electrodynamic force has a strong stabilizing influence in the symmetric mode and can suppress the gravitational instability above a certain value of the basic electric field.

PACS numbers: 47.55.Cy 


\section{Introduction}

Since the turn of the century it has been known that circular static inviscid fluid jets are capillary unstable to axisymmetric mode of disturbances having small axial wave numbers. Observations have shown that this capillary instability, driven by surface forces on the jet interface, leads to the break up of the jet into droplets.

In the present time, particularly in the last few decades, the hydrodynamic, magnetohydrodynamic and electrodynamic stability of numerous cylindrical (or other) configurations has received the attention of many investigators [1-8]. Their results have been essential not only due to their practical applications ranging from the design of sprays to the design of ink-jet printers but also for their crucial applications in several fields of science, e.g. industrial field, geophysics, astrophysics, etc.

Chandrasekhar and Fermi $[9,10]$ elaborated the related problem of a fluid jet acting on its own attraction to small axisymmetric disturbances. Such a study has a correlation with understanding the dynamical behaviour of the spiral arms of galaxies (cf. [9]). Radwan [11] has recently modified the stability criterion of [9] and [10] by including the effect of the electrodynamic force on the gravitational fluid pervaded by a homogeneous and uniform electric field.

The main aim of the present work is to investigate the electrodynamic instability of a self-gravitating fluid cylinder embedded in a self-gravitating vacuum under generally varying electric field. The recent results of one of us [11] can be recovered as a limiting case from the present analysis.

\section{Formulation of the problem}

Consider a dielectric gravitational fluid cylinder of dielectric constant $\varepsilon^{(i)}$ ambient with dielectric self-gravitating vacuum of dielectric constant $\varepsilon^{(e)}$. The fluid is assumed to be homogeneous, incompressible, non-viscous and with uniform density $\rho$. The superscripts (i) and (e) characterize the variables interior and exterior to the fluid cylinder. We assume that the quasi static approximation (cf. [5] and [12]), is valid for the considered problem. In the basic state it is proposed that there are no surface charges at the fluid boundary surface and consequently the surface charge density will be considered to be zero during the perturbation [5]. The fluid is acting up on the self-gravitating, inertia and electrodynamic forces. In the regions surrounding the fluid, the only force existing there is the electrodynamic force. We shall use the cylindrical polar coordinates $(r, \phi, z)$ system with the $z$-axis coinciding with the axis of the fluid cylinder.

The basic equations for such a problem under consideration are the combination of the pure hydrodynamic, self-gravitating and Maxwell's equations. Under the present circumstances they are as follows:

$$
\begin{aligned}
& \rho^{(\mathrm{i})}\left(\frac{\partial u^{(\mathrm{i})}}{\partial t}+\left(u^{(\mathrm{i})} \cdot \nabla\right) u^{(\mathrm{i})}\right)=\frac{1}{2} \nabla\left[\varepsilon^{(\mathrm{i})}\left(\boldsymbol{E}^{(\mathrm{i})} \cdot \boldsymbol{E}^{(\mathrm{i})}\right)\right] \\
& \quad-\nabla P^{(\mathrm{i})}-\rho(\mathrm{i}) \nabla V^{(\mathrm{i})}, \\
& \operatorname{div} u^{(\mathrm{i})}=0
\end{aligned}
$$




$$
\begin{aligned}
& \operatorname{div}(\varepsilon E)^{(\mathrm{i}),(\mathrm{e})}=0, \\
& \operatorname{curl} E^{(\mathrm{i}),(\mathrm{e})}=0, \\
& \nabla^{2} V^{(\mathrm{i})}=-4 \pi G \rho^{(\mathrm{i})}, \\
& \nabla^{2} V^{(\mathrm{e})}=0 .
\end{aligned}
$$

IIere $\rho^{(i)}, u^{(i)}$ and $P^{(i)}$ are the fluid mass density, velocity vector and kinetic pressure, respectively, $G$ is the gravitational constant, $V^{(\mathrm{e})}$ and $V^{(\mathrm{i})}$ are the gravitational potentials exterior and interior of the fluid cylinder, $E^{(e)}$ and $E^{(i)}$ are the electric field intensities acting outside and inside the fluid cylinder, respectively.

The basic state corresponding to $u=u_{0}=0$ is studied in detail and the fundamental quantities in the equilibrium state are identified.

Assuming the dielectric fluid cylinder is pervaded by the longitudinal uniform electric field $\boldsymbol{E}_{0}^{(i)}=\left(0,0, E_{0}\right)$, the basic equations (3) and (4) are solved in such a state and we obtain

$$
\boldsymbol{E}_{0}^{(e)}=\left(0, \beta a r^{-1}, \alpha\right) \boldsymbol{E}_{0},
$$

where $\alpha$ and $\beta$ are the exterior electric field parameters. The gravitational equations (5) and (6) are solved and the final forms of $V_{0}^{(\mathrm{i})}$ and $V_{0}^{(\mathrm{e})}$ are given by:

$$
\begin{aligned}
& V_{0}^{(\mathrm{i})}=-\pi G \rho^{(\mathrm{i})} r^{2}, \\
& V_{0}^{(\mathrm{e})}=\pi G \rho^{(\mathrm{i})} a^{2}\left[\log \left(\frac{a}{r}\right)^{2}-1\right],
\end{aligned}
$$

where the subscripts 0 will characterize the equilibrium quantities and later the quantities with index 1 will be the fluctuation quantities. Solving Eqs. (1) and (2) and utilizing Eqs. (7)-(9), the fluid hydrostatic pressure is identified and given by:

$$
P_{0}=\pi G\left(\rho^{(\mathrm{i})}\right)^{2}\left(a^{2}-r^{2}\right)+\left(E_{0}^{2} / 2\right)\left[\varepsilon^{(\mathrm{i})}-\varepsilon^{(e)}\left(\alpha^{2}+\beta^{2}\right)\right] .
$$

It is clear from (10) at the fluid boundary surface $r=a$, the restrictions

$$
\varepsilon^{(\mathrm{i})} \geq \varepsilon^{(\mathrm{e})}\left(\alpha^{2}+\beta^{2}\right)
$$

must be satisfied, where the cquality holds a limiting case with zero fluid pressure. If the self-gravitating medium surrounding the cylinder has the same permittivity constant as that of the fluid, the conditions are given explicitly by:

$$
\alpha^{2}+\beta^{2} \leq 1
$$

\section{Perturbation analysis}

Let the basic state be perturbed, then for small departures from the equilibrium state every perturbed quantity $Q(r, \phi, z ; t)$ can be expanded as

$$
Q(r, \phi, z ; \ell)=Q_{0}+\delta(\ell) Q_{1}(r, \phi, z) .
$$

IIere $Q$ stands for each of $u, P, E^{(\mathrm{i}),(\mathrm{e})}, V^{(\mathrm{i}),(\mathrm{e})}$ and the perturbed radial distance of the fluid cylinder, where the quantities with index 1 are the fluctuation part of $Q$. The amplitude $\delta(t)$ of the perturbed wave surface at any instant of time $t$ is being:

$$
\delta(t)=\delta_{0} \exp (\sigma t),
$$


where $\sigma$ is the temporal amplification and $\delta_{0}$ is the initial amplitude.

In view of Eqs. (13) and (14), and in considering the influence of a small wave disturbance to the boundary interface $r=a$ propagating in the positive $z$-direction, the surface deflection is being:

$$
r=a+\eta
$$

with

$$
\eta=\delta(t) a \exp [\mathrm{i}(k z+m \phi] .
$$

IIere $\eta$ is the elevation of the perturbed surface wave measured from the equilibrium position and normalized with respect to $a$ and $k$ (any real number) is the longitudinal wave number and $m$ (an integer) is the transverse wave number. by:

By means of Eqs. (13)-(16), the relevant perturbation equations are given

$$
\begin{aligned}
& \sigma u_{1}^{(\mathrm{i})}=-\nabla \Pi_{1}^{(\mathrm{i})}, \\
& \operatorname{div} u_{1}^{(\mathrm{i})}=0, \\
& \operatorname{div}\left(\varepsilon E_{1}\right)^{(\mathrm{i}),(\mathrm{e})}=0, \\
& \operatorname{curl} \boldsymbol{E}_{1}^{(\mathrm{i}),(\mathrm{e})}=0, \\
& \nabla^{2} V_{1}^{(\mathrm{i}),(\mathrm{e})}=0,
\end{aligned}
$$

where

$$
\Pi_{1}^{(\mathrm{i})}=\left(P_{1}^{(\mathrm{i})} / \rho^{(\mathrm{i})}\right)-V_{1}^{(\mathrm{i})}-\left(\varepsilon^{(\mathrm{i})} / 2 \rho^{(\mathrm{i})}\right)(\boldsymbol{E} \cdot \boldsymbol{E})_{1} .
$$

Taking the divergence of the vector equation of motion (17) and utilizing Eq. (18), we get

$$
\nabla^{2} \Pi_{1}^{(\mathrm{i})}=0 \text {. }
$$

The circulation equation (20) concerning the perturbed electric field intensities interior and exterior to the fluid cylinder means that $\boldsymbol{E}_{1}^{(\mathrm{i}),(\mathrm{e})}$ can be derived by means of scalar functions:

$$
\boldsymbol{E}_{1}^{(\mathrm{i}),(\mathrm{e})}=-\nabla \Psi_{1}^{(\mathrm{i}),(\mathrm{e})} .
$$

Inserting Eq. (24) into Eq. (19) one yields

$$
\nabla^{2} \Psi_{1}^{(\mathrm{i}),(\mathrm{e})}=0 \text {. }
$$

Therefore the solution of the relevant perturbation (17)-(22) can be obtained by solving Laplace's Eqs. (21), (23) and (25). In view of the $(z, \phi)$-dependence given by Eq. (16) and based on the linear perturbation technique, the scalar functions $\Pi_{1}^{(\mathrm{i})}, \Psi_{1}^{(\mathrm{i}),(\mathrm{e})}$ and $V_{1}^{(\mathrm{i}),(\mathrm{e})}$ can be written as $\exp [\sigma t+\mathrm{i}(k z+m \phi)]$ times an amplitude function of $r$. From this point of view, Eqs. (21), (23) and (25) are solved and the infinite solutions are excluded. The non-singular solutions are given by:

$$
\begin{aligned}
& \Pi_{1}^{(\mathrm{i})}=A \eta I_{m}(k r), \\
& V_{1}^{(\mathrm{i})}=B \eta I_{m}(k r),
\end{aligned}
$$




$$
\begin{aligned}
& V_{1}^{(\mathrm{e})}=\tilde{B} \eta K_{m}(k r), \\
& \Psi_{1}^{(\mathrm{i})}=C \eta I_{m}(k r), \\
& \Psi_{1}^{(\mathrm{e})}=\tilde{C} \eta K_{m}(k r),
\end{aligned}
$$

where $I_{m}(k r)$ and $K_{m}(k r)$ are the modified Bessel functions of the first and second kind of the order $m$, and $A, B, \tilde{B}, C$ and $\tilde{C}$ are constants of integration to be determined.

\section{Boundary conditions and dispersion relation}

The solutions (26)-(30) of the relevant perturbation Eqs. (17)-(22) must satisfy certain boundary conditions. For the problem at hand these appropriate boundary conditions at $r=a$ are the following:

(i) The normal component of the velocity must be compatible with the velocity of the perturbed boundary surface across the interface (15).

(ii) The gravitational potential $V=v_{0}+\delta v_{1}$ and its derivative are continuous across the perturbed boundary surface (15).

(iii) The electric potential $\Psi$ must be continuous across the perturbed interface $r=a+\eta$.

(iv) The normal component of the electric displacement must also be continuous across the perturbed boundary surface (15).

(v) Finally, the normal componcnt of the total stress tensor must also be continuous across the perturbed surface $r=a+\eta$.

Afterwards, we will obtain the analytic expressions of dispersion relations, solving algebraically Eqs. (7), (16), (24), (26)-(30) on the above boundary conditions.

The dispersion relations are as follows:

$$
\begin{aligned}
& \sigma^{2}=4 \pi G \rho^{(\mathrm{i})}\left(x I_{m}^{\prime}(x) / I_{m}(x)\right)\left[I_{m}(x) K_{m}(x)-\frac{1}{2}\right] \\
& -\frac{\varepsilon^{(\mathrm{e})} E_{0}^{2}}{\rho^{(\mathrm{i})} a^{2}}\left\{\beta^{2} \frac{x I_{m}^{\prime}(x)}{I_{m}(x)}+\left[x \varepsilon^{(\mathrm{i})}(m \beta+\alpha x)-\varepsilon^{(\mathrm{e})}(m \beta+\alpha x)^{2}\right] L_{m}(x)\right\}
\end{aligned}
$$

where

$$
L_{m}(x)=I_{m}^{\prime}(x) K_{m}(x)\left[\varepsilon^{(\mathrm{e})} I_{m}(x) K_{m}^{\prime}(x)-\varepsilon^{(\mathrm{i})} I_{m}^{\prime}(x) K_{m}(x)\right]^{-1},
$$

and $x=k a$ is the dimensionless longitudinal wave number.

Equation (31) is the desired eigenvalue relation of an electrodynamic gravitational fluid cylinder ambient with self-gravitating vacuum that pervaded by generally varying electric field. By means of the stability criterion (31) the ordinary stability states as well as those of instability can be identified. The points at which a transition from stability to instability states and vice versa (i.e. the marginal stability) can be also determined by just putting $\sigma=0$ in the dispersion equation (31). The characteristic equation (31) relates the temporal amplification $\sigma$ or rather the oscillation frequency $\omega$ (that if $\sigma=\mathrm{i} \omega$ is imaginary) with the modified Bessel functions of the first and second kind of the order $m$ and their 
derivatives, the fundamental quantities $\left(4 \pi G \rho^{(\mathrm{i})}\right)^{-1 / 2}$ and $\left(\varepsilon^{(e)} E_{0}^{2} / \rho^{(\mathrm{i})} a^{2}\right)^{-1 / 2}$ as a unit of time, the wave numbers $x$ and $m$, the dielectric constants $\varepsilon^{(\mathrm{i})}$ and $\varepsilon^{(\mathrm{e})}$, the scale length $a$ of the geometric figure and with the parameters $\alpha, \beta$ of the vacuum varying electric field.

The eigenvalue relation (31) is a simple linear combination of the eigenvalue relations of a full fluid cylinder surrounded by a vacuum being subjected to the self-gravitating force only and that dielectric one acted on the electrodynamic force only. This simple additivity is not only due to the linearization of the fundamental equations (1)-(6) or the simple form of the electric field equations (3) and (4) but also because no volume and no surface charges are assumed to be present in the bulk and at the interface of the fluid cylinder. Indeed it is found that this additivity is true whether the electric field is uniform [11] or not.

\section{Gravitational instability}

Neglecting the influence of the electrodynamic force, the eigenvalue relation (31) yields

$$
\sigma^{2} /\left(4 \pi G \rho^{(\mathrm{i})}\right)=\left[x I_{m}^{\prime}(x) / I_{m}(x)\right]\left(I_{m}(x) K_{m}(x)-\frac{1}{2}\right) .
$$

This relation is valid for all axisymmetric $m=0$ and non-axisymmetric $m \geq 1$ modes of perturbation for all (short and long) wavelengths. In the rotationally symmetric (sausage) mode $m=0$, the dispersion relation (32) reduces to

$$
\sigma^{2}=4 \pi G \rho^{(\mathrm{i})}\left[x I_{1}(x) / I_{0}(x)\right]\left(I_{0}(x) K_{0}(x)-\frac{1}{2}\right) .
$$

The relation (33) was derived for the first time by Chandrasekhar and Fermi [9] by employing the principle of conservation of energy.

By a resort to the recurrence relations of the modified Bessel functions

$$
2 L_{m}^{\prime}(x)=L_{m-1}(x)+L_{m+1}(x),
$$

where $L_{m}^{\prime}(x)$ stands for $I_{m}^{\prime}(x)$ and $K_{m}^{\prime}(x)$, and using the fact for $x \neq 0$ that $I_{m}(x)$ and $K_{m}(x)$ are always positive, we can prove that $I_{m}^{\prime}(x)$ is always positive while $K_{m}^{\prime}(x)$ is never positive. Hence for $x \neq 0$, we have

$$
\left[x I_{m}^{\prime}(x) / I_{m}(x)\right]>0 \text {. }
$$

Consequently the sign of $\sigma^{2} /\left(4 \pi G \rho^{(\mathrm{i})}\right)$ can be determined by identifying the sign of the quantity $\left(I_{m}(x) K_{m}(x)-1 / 2\right)$ for all $x \neq 0$ values in all possible modes of perturbations. Since from the propertics of the modified Bessel functions,

$$
2 I_{m}(x) K_{m}(x)<1
$$

for each non-zero real value of $x$ in all non-axisymmetric modes $m \geq 1$ of perturbation. Therefore the fluid cylinder is self-gravitating stable to all purely non-axisymmetric modes $m \neq 0$ ( $m$ is integer) of disturbance for all (short and long) wavelengths.

To the rotationally axisymmetric mode $m=0$, the fluid cylinder is gravitationally unstable for $x<1.0668$ only, while it is gravitationally stable for $x \geq 1.0668$, where the equality holds for the marginal (neutral) stability. 
For more details about the stability and instability domains and regions of such a case, we may refer to [9].

\section{Electrofluid dynamic instability}

Neglecting the self-gravitating force influence assuming the electrodynamic effect is paramount over that of the gravitational force, the dispersion equation (31) degenerates to

$$
\sigma^{2}=\frac{\varepsilon^{(\mathrm{e})} E_{0}^{2}}{\rho^{(\mathrm{i}) a^{2}}}\left\{(m \beta+x \alpha)\left[\varepsilon^{(\mathrm{e})}(m \beta+x \alpha)-\varepsilon^{(\mathrm{e})} x\right] L_{m}(x)-\beta^{2} \frac{x I_{m}^{\prime}(x)}{I_{m}(x)}\right\} .
$$

In view of the properties of the modified Bessel functions, assuming that $\varepsilon^{(e)}$ and $\varepsilon^{(i)}$ are positive definite, and by an appeal to the recurrence relations (34), we can prove that

$$
L_{m}(x)<0
$$

for all $x \neq 0$ and $m \geq 0$ values. Also, in view of the fact that $I_{m}(x)$ is always positive and monotonically positive together with the recurrence relation (34) one can show that

$$
\left[x I_{m}^{\prime}(x) / I_{m}(x)\right]>0
$$

for each non-zero real value of $x$ to all possible modes $m \geq 0$ of disturbance.

Now returning to the stability criterion (37) and assuming that the present model is acting upon the uniform vacuum clectric field $\left(0,0, \alpha E_{0}\right)$, i.e. $\beta=0$, we have

$$
\sigma^{2} /\left(\varepsilon^{(\mathrm{e})} E_{0}^{2} / \rho^{(\mathrm{i})} a^{2}\right)=\alpha x^{2}\left(\alpha \varepsilon^{(\mathrm{e})}-\varepsilon^{(\mathrm{i})}\right) L_{m}(x) .
$$

From the analytical discussions of the relation (39), we conclude that the dielectric fluid cylinder is electrodynamic stable if $\alpha \varepsilon^{(\mathrm{e})}>\varepsilon^{(\mathrm{i})}$ for any value of $\alpha$ different from zero for all $m \geq 0$ and $x \neq 0$ values.

If the model under consideration is acting on the vacuum varying electric field $\left(0, \beta E_{0} a / r, 0\right)$, i.e. $\alpha=0$, the relation (37) is reduced to the non-dimensional form

$$
\frac{\sigma^{2}}{\left(\varepsilon^{(\mathrm{e})} E_{0} / \rho^{(\mathrm{i})} a^{2}\right)}=m \beta\left(m \beta \varepsilon^{(\mathrm{e})}-x \varepsilon^{(\mathrm{i})}\right) L_{m}(x)-\beta^{2}\left(x I_{m}^{\prime}(x) / I_{m}(x)\right) .
$$

For axisymmetric disturbance $m=0$, the dielectric fluid cylinder is stable or unstable if $\beta^{2}\left(x I_{m}^{\prime}(x) / I_{m}(x)\right)$ is positive or negative, repectively. The sign of $\beta^{2}$ is immaterial, while $\left(x I_{0}^{\prime}(x) / I_{0}(x)\right)$ is always positive for each non-zero real value of $x$. Therefore, we deduce, in the case of $m=0$, that the dielectric cylinder is electrodynamic stable for all (short and long) wavelengths whatever is the value of $\beta$.

For non-axisymmetric modes $m \geq 1$, the electrodynamic force has stabilizing influence in the terms $\beta^{2}\left(x I_{m}^{\prime}(x) / I_{m}(x)\right)$ and $\varepsilon^{(\mathrm{e})}(m \beta)^{2} L_{m}(x)$ while it is destabilizing in the term $\varepsilon^{(i)} m \beta x L_{m}(x)$. Therefore, we predict, when $m \geq 1$, that the dielectric cylinder is electrodynamic stable for all $x \neq 0$ values if the restrictions

$$
\left.\beta\left\{\varepsilon^{(\mathrm{e})} m^{2} L_{m}(x)+\left[x I_{m}^{\prime}(x) / I_{m}(x)\right)\right]\right\} \geq \varepsilon^{(\mathrm{i})} m x L_{m}(x),
$$


and vice versa where the equality corresponding to the marginal stability.

Now combining the (in-) stability results of the stability criteria (39) and (40), one can find out the (in-) stability conclusions of the general relation (37) concerning the dielectric fluid cylinder acting on the electrodynamic force only. As a general conclusion one can say that there will be ordinary stable and ordinary unstable domains which can be determined according to some restrictions. Moreover, the neutral (marginal) stability occurs if the restriction:

$$
\varepsilon^{(\mathrm{e})}(m \beta+\alpha x)^{2} L_{m}(x)+\beta^{2}\left[x I_{m}^{\prime}(x) / I_{m}(x)\right]=\varepsilon^{(\mathrm{i})} x(m \beta+\sigma x) L_{m}(x)
$$

is satisfied.

\section{Electro-gravitodynamic instability}

In such a general case the dielectric self-gravitating fluid cylinder surrounded by a dielectric self-gravitating vacuum is acting on the combined effect of the gravitational and electrodynamic forces. It is more convenient to rewrite the eigenvalue relation (31) in the following non-dimensional form:

$$
\begin{aligned}
& \sigma^{2} /\left(4 \pi G \rho^{(\mathrm{i})}\right)=\left[I_{m}(x) K_{m}(x)-\frac{1}{2}\right]\left[x I_{m}^{\prime}(x) / I_{m}(x)\right]+\left(E_{0} / E_{G}\right)^{2} \\
& \times\left\{(m \beta+\alpha x)\left[\varepsilon^{(\mathrm{e})}(m \beta+\alpha x)-\varepsilon^{(\mathrm{i})} x\right] L_{m}(x)-\beta^{2}\left[x I_{m}^{\prime}(x) / I_{m}(x)\right]\right\},
\end{aligned}
$$

where

$$
E_{G}=2 a \rho^{(\mathrm{i})}\left(\pi G / \varepsilon^{(\mathrm{e})}\right)^{1 / 2} .
$$

Combining the results of the above different sections, the electro-gravitodynamic stability results of the present general case can be identified.

In the non-axisymmetric modes $m \geq 1$ of perturbations, it is found that the electrodynamic force has a stabilizing or destabilizing influence according to some restrictions. Since the model is gravitationally stable, the electrodynamic force has the feature of increasing or decreasing such stability according to some conditions. Ilowever the predominating character of the electrodynamic force is the stabilizing influence. Hence we predict that the electrodynamic force has the power to suppress the gravitational instability and this will be very clear below in the most dangerous (sausage) mode $m=0$ of perturbation.

In the rotationally axisymmetric (sausage) mode $m=0$ of disturbance, it is found that the marginal stability occurred as $\sigma^{2} /\left(4 \pi G \rho^{(\mathrm{i})}\right)=0$ and consequently the model will be completely stable as long as

$$
\begin{aligned}
& \left(E_{0} / E_{G}\right)^{2} \geq\left[I_{0}(x) K_{0}(x)-\frac{1}{2}\right]\left\{\beta^{2}+\alpha\left(\varepsilon^{(\mathrm{e})}-\alpha \varepsilon^{(\mathrm{e})}\right)\right. \\
& \left.\quad \times\left[x I_{0}(x) L_{0}(x) / I_{1}(x)\right]\right\}^{-1}
\end{aligned}
$$

where the equality is corresponding to the neutral stability.

For suitable values of $\alpha, \beta, \varepsilon^{(\mathrm{i})}$ and $\varepsilon^{(\mathrm{e})}$ (see the inequality (11)) one can determine exactly the value of $\left(E_{0} / E_{G}\right)$ above which the gravitational instability is completely suppressed and then stability arrises. This can be carried out and completed by introducing the general eigenvalue (43) in the computer and also to determine the stability states and those of instability as well. 


\section{Refercnces}

[1] L. Baker, Phys. Fluids 26, 391 (1983).

[2] S. Chandrasekhar, Hydrodynamic and IIydromagnetic Stability, Dover, New York 1981.

[3] N.T. El Dabe, E.F. El Shehawey, G.M. Moatimid, A.A. Mohamed, J. Math. Phys. 26, 2072 (1985).

[4] J.M. Kendall, Phys. Fluids 29, 2086 (1986).

[5] A.A. Mohamed, A.G. El-Sakka, G.M. Sultan, Physica Scr. 31, 193 (1985).

[6] M. Tajiri, J. Phys. Soc. Jpn. 53, 3759 (1984).

[7] A.E. Radwan, S.S. Elazab, Simon Stevin 61, 293 (1987); J. Phys. Soc. Jpn. 57, 461 (1988); ibid. 58, 155 (1989).

[8] D.K. Callabaut, A.E. Radwan, Proc. Europ. Phys. Soc. D 10, 11 (1986).

[9] S. Chandrasekhar, E. Fermi, Astrophys. J. 118, 116 (1953).

[10] S. Chandrasekhar, Proc. Naln. Acad. Sci. (USA) 46, 453 (1960).

[11] A.E. Radwan, J. Phys. Soc. Jpn. 58, 1273 (1989).

[12] H.H. Woodson, J.R. Melcher, Electromechanical Dynamics, Wiley, New York 1968. 